See discussions, stats, and author profiles for this publication at: https://www.researchgate.net/publication/265297708

\title{
Circulant Games
}

Article in Theory and Decision · November 2014

DOI: 10.1007/s11238-014-9478-4

2 authors, including:

(3.) Georg D. Granic

(1.) Erasmus University Rotterdam 14 PUBLICATIONS 38 CITATIONS

SEE PROFILE 


\title{
Circulant Games
}

\section{Đura-Georg Granić* Johannes Kern ${ }^{\dagger}$}

September 4, 2014

\begin{abstract}
We study a class of two-player normal-form games with cyclical payoff structures. A game is called circulant if both players' payoff matrices fulfill a rotational symmetry condition. The class of circulant games contains well-known examples such as Matching Pennies, Rock-Paper-Scissors, as well as subclasses of coordination and common interest games. The best response correspondences in circulant games induce a partition on each player's set of pure strategies into equivalence classes. In any Nash Equilibrium all strategies within one class are either played with strictly positive or with zero probability. We further show that, strikingly, a single parameter fully determines the exact number and the structure of all Nash equilibria (pure and mixed) in these games. The parameter itself only depends on the position of the largest payoff in the first row of one of the player's payoff matrix.
\end{abstract}

JEL-Classification: C70, C72, D00

Keywords: Bimatrix Games, Circulant Games, Circulant Matrix, Number of Nash Equilibria, Rock-Paper-Scissors

Working Paper. This is an author-generated version of a research manuscript which is currently under evaluation at a journal. It is circulated exclusively for the purpose of facilitating scientific discussion. The final version of the article might differ from this one.

\footnotetext{
*Department of Economics, University of Cologne, 50923 Cologne (Germany), georg.granic@uni-koeln.de
}

${ }^{\dagger}$ Department of Economics, University of Cologne, 50923 Cologne (Germany), johannes.kern@uni-koeln.de 


\section{Introduction}

Games with cyclical structures are ubiquitous in game theory. Simple examples like Matching Pennies and Rock-Paper-Scissors are routinely used to illustrate the concepts of mixed strategies and mixed strategy Nash equilibria in any introductory class to game theory. Beyond their pedagogical value, these simple examples have a wide range of application in game theory. Evolutionary game theory is one prominent example and, e.g., the mating strategies of the common side-blotched lizard have been shown to follow a rock-paper-scissors pattern (Sinervo and Lively, 1996). A cyclical game structure can be captured by circulant payoff matrices, in which each row vector is rotated by one element relative to the preceding row vector (Hofbauer, Schuster, Sigmund, and Wolff, 1980; Diekmann and van Gils, 2009). Games with circulant payoff matrices have been studied extensively in the literature on evolutionary game theory (Hofbauer and Sigmund, 1998) and population dynamics (Hofbauer, Schuster, Sigmund, and Wolff, 1980; Diekmann and van Gils, 2009). Circulant payoff matrices also underly certain classes of coordination games, starting with matching games, that have been studied in the literature on symmetries and focal points (Casajus, 2000; Janssen, 2001). ${ }^{1}$

The class of games we study here is important for at least two fields of applications. First, the analysis of the convergence properties of various evolutionary dynamics for cyclical game structures has often focused on uniformly mixed strategies. Games in which this strategy profile is the unique equilibrium constitute important examples of convergence failure (see, e.g., Sandholm, 2010, Chapter 9.2.1, pp. 327-330). Still, many games with a cyclical structure have more than one equilibrium and the non-convergence to one particular equilibrium may not be conclusive for the convergence properties of the whole system. Second, matching games and more general coordination games constitute an archetypal framework to analyze features external to the games' formal structure. The cyclical game structure provides a framework where strategies cannot be differentiated according to differences in payoffs. Yet, matching games are just one particular representation of such symmetric frameworks and many different, equally appropriate cyclical game structures may exist (see, e.g., Alós-Ferrer and Kuzmics, 2013). A rigorous characterization of the set of Nash equilibria of cyclical game structures in general is still missing.

The aim of this paper is to bridge these gaps and provide a more general analysis of games with a cyclical structure. More precisely, we investigate a class of finite two-player normal-form $n \times n$ games we coin circulant games, in which the players' payoff matrices are circulant. We also require that the first row of each matrix is ordered. This approach allows us to integrate classical examples from Game Theory into one single class of games. Well-known games such as the ones mentioned above, as well as subclasses of common-interest and coordination games (including matching games) belong to the class of circulant games.

Our results shed new light on the common features shared by these games. Our main results identify the exact number of (pure or mixed) Nash equilibria in circulant games. We also obtain necessary and sufficient conditions for the existence of pure strategy Nash equilibria and, in case of non-existence, for the uniqueness of the uniformly mixed Nash equilibrium (a profile which we show to be a Nash equilibrium for all circulant games). As a consequence of our main results we obtain that the maximal number of Nash equilibria in these games is exactly $2^{n}-1$. The number of pure strategy Nash equilibria is either $0,1,2$, or $n$. Further, we are also able to characterize the structure of the set of mixed Nash equilibria. The best response correspondences induce an equivalence relation on each player's set of pure strategies. In any Nash equilibrium all strategies within one equivalence class are either played with strictly

\footnotetext{
${ }^{1}$ The simplest example of a matching game is Heads and Tails. If both players match the strategy of the other player each player gets a payoff of 1, otherwise each player receives a payoff of zero.
} 
positive or with zero probability. We show how to derive the equivalence classes, allowing for a characterization of the support of all Nash equilibrium strategies.

Our results also contribute to the literature on the number of Nash equilibria in finite twoplayer normal-form $n \times n$ games. Provided that such a game is non-degenerate the number of Nash equilibria is finite and odd (see, e.g., Shapley, 1974). Quint and Shubik (1997) show that for any odd integer number $y$ between 1 and $2^{n}-1$, there exists a game with exactly $y$ Nash equilibria. However, as shown in von Stengel (1997), $2^{n}-1$ is not an upper bound on the number of Nash equilibria in such games. New upper bounds on the number of distinct Nash equilibria are established in Keiding (1998) and von Stengel (1999). For the class of coordination games $2^{n}-1$ is the (tight) upper bound on the number of equilibria (Quint and Shubik, 2002). Our results show that this is also true for the class of circulant games.

Recently, several other articles have analyzed subclasses of games with a special focus on different notions of cyclicity. Duersch, Oechssler, and Schipper (2012) consider symmetric twoplayer zero-sum normal-form games and define generalized rock-paper-scissors matrices $(g R P S)$ in terms of best response cycles. In their setting, a game has a pure strategy Nash equilibrium if and only if it is not a $g R P S$. Bahel (2012) and Bahel and Haller (2013) examine zero-sum games that are based on cyclic preference relations on the set of actions and characterize the set of Nash equilibria. In the former paper, actions are distinguishable, i.e., one specific actions is the beginning of the cyclic relation, and there exists a unique Nash equilibrium. In the latter, actions are anonymous, i.e., each action can be seen as the beginning of the cycle without affecting the relation, and depending on the number of actions the Nash equilibrium is unique or there exists an infinite number of Nash equilibria.

The remainder of this paper is structured as follows. Section 2 introduces the class of circulant games. Section 3 states the main results and presents a recipe to characterize the support of all Nash equilibrium strategies for a given circulant game. Section 4 presents generalizations of circulant games and Section 5 concludes. All proofs are relegated to the appendix.

\section{Circulant Games}

Let $\Gamma=\left(\left(S_{1}, S_{2}\right),\left(\pi_{1}, \pi_{2}\right)\right)$ be a finite two-player normal-form game where $S_{i}=\left\{0,1, \ldots, n_{i}-1\right\}$ denotes player $i$ 's set of pure strategies and $\pi_{i}: S_{1} \times S_{2} \rightarrow \mathbb{R}$ denotes player $i$ 's payoff function for $i=1,2 .{ }^{2}$ We will write player $i$ 's payoff function as the $n_{1} \times n_{2}$ matrix $A_{i}=\left(a_{k l}^{i}\right)_{k \in S_{1}, l \in S_{2}}$ given by $a_{k l}^{i}=\pi_{i}(k, l)$. Thus in both matrices each row corresponds to a pure strategy of player 1 and each column to a pure strategy of player 2. Following the notation in e.g. Alós-Ferrer and Kuzmics (2013), we will also write $\pi_{i}\left(s \mid s^{\prime}\right)$ for player $i$ 's payoff if he chooses a strategy $s$ and player $-i$ chooses strategy $s^{\prime}$. The set of mixed strategies for player $i$ is denoted by $\Sigma_{i}$. For $\sigma_{i} \in \Sigma_{i}, \sigma_{i}(s)$ denotes the probability that $\sigma_{i}$ places on the pure strategy $s \in S_{i}$. The set of all pure strategies played with strictly positive probability is denoted by $\operatorname{supp}\left(\sigma_{i}\right)$. Payoff functions are extended to the sets of mixed strategies through expected payoffs. Given a mixed strategy $\sigma_{-i}$ of player $-i$, a best response for player $i$ against $\sigma_{-i}$ is a strategy $\sigma_{i}$ such that $\pi_{i}\left(\sigma_{i} \mid \sigma_{-i}\right) \geq \pi_{i}\left(\sigma_{i}^{\prime} \mid \sigma_{-i}\right)$ for all $\sigma_{i}^{\prime} \in \Sigma_{i}$. The set of best responses for player $i$ against a strategy $\sigma_{-i}$ of the other player is denoted by $B R_{i}\left(\sigma_{-i}\right)$. A finite two-player normal-form game is non-degenerate (Quint and Shubik, 1997) if for any mixed strategy $\sigma_{i}$ of player $i$ with $\left|\operatorname{supp}\left(\sigma_{i}\right)\right|=m$, player $-i$ has at most $m$ pure strategy best responses against $\sigma_{i}$. In what follows $\Gamma_{n}$ denotes a finite two-player normal-form game in which $S_{1}=S_{2}=S^{n}=\{0, \ldots, n-1\}$.

The following two results are well-known and will be used throughout the paper.

\footnotetext{
${ }^{2}$ We choose to label players' strategies from 0 to $n_{i}-1$ as this will later simplify notation significantly.
} 
Proposition 1 (Best Response Condition, Nash, 1951). Let $\Gamma$ be a finite two-player normalform game. Then $\sigma_{i} \in \Sigma_{i}$ is a best response to $\sigma_{-i} \in \Sigma_{-i}$ if and only if for all $s_{i} \in S_{i}$

$$
\sigma_{i}\left(s_{i}\right)>0 \Rightarrow \pi_{i}\left(s_{i} \mid \sigma_{-i}\right)=\max _{s \in S_{i}} \pi_{i}\left(s \mid \sigma_{-i}\right) .
$$

Proposition 2 (Shapley, 1974; Quint and Shubik, 1997). Let $\Gamma$ be a finite non-degenerate two-player normal-form game with strategy set $S_{1}=S_{2}=S$. Then

(i) $\Gamma$ has a finite and odd number of Nash equilibria.

(ii) if $T_{1}, T_{2} \subseteq S$ then $\Gamma$ has at most one Nash equilibrium $\left(\sigma_{1}, \sigma_{2}\right)$ such that $\operatorname{supp}\left(\sigma_{1}\right)=T_{1}$ and $\operatorname{supp}\left(\sigma_{2}\right)=T_{2}$.

Circulant games will be defined through circulant matrices (see Davis, 1979) which we introduce now.

Definition 1. A matrix $A \in \mathbb{R}^{n \times n}$ is circulant if it has the form

$$
A=\left(\begin{array}{ccccc}
a_{0} & a_{1} & a_{2} & \cdots & a_{n-1} \\
a_{n-1} & a_{0} & a_{1} & \ldots & a_{n-2} \\
a_{n-2} & a_{n-1} & a_{0} & \ldots & a_{n-3} \\
\vdots & \vdots & \vdots & \ddots & \vdots \\
a_{1} & a_{2} & a_{3} & \cdots & a_{0}
\end{array}\right)
$$

and anti-circulant if

$$
A=\left(\begin{array}{ccccc}
a_{0} & \cdots & a_{n-3} & a_{n-2} & a_{n-1} \\
a_{1} & \cdots & a_{n-2} & a_{n-1} & a_{0} \\
a_{2} & \cdots & a_{n-1} & a_{0} & a_{1} \\
\vdots & . & \vdots & \vdots & \vdots \\
a_{n-1} & \cdots & a_{n-4} & a_{n-3} & a_{n-2}
\end{array}\right) .
$$

Circulant and anti-circulant matrices are hence fully specified by the first row vector. Each remaining row vector is rotated by one element relative to the preceding row vector. We are now ready to define a circulant game.

Definition 2. A two-player normal-form game $\Gamma_{n}$ is a circulant game if

(i) each player's payoff matrix is either circulant or anti-circulant,

(ii) $a_{0}^{1}>a_{1}^{1} \geq \cdots \geq a_{n-1}^{1}$, and

(iii) either $a_{n-k}^{2}>a_{n-k+1}^{2} \geq \cdots \geq a_{n-1}^{2} \geq a_{0}^{2} \geq a_{1}^{2} \geq \cdots \geq a_{n-k-1}^{2}$ or $a_{n-k}^{2}>a_{n-k-1}^{2} \geq \cdots \geq$ $a_{1}^{2} \geq a_{0}^{2} \geq a_{n-1}^{2} \geq \cdots \geq a_{n-k+1}^{2}$ for some $1 \leq k \leq n$.

The parameter $k$ is called the shift of $\Gamma_{n}$.

The shift describes the position of player 2's largest payoff in the first row of his payoff matrix. As we will see later, knowing the shift and the number of pure strategies suffices to determine the exact number and structure of Nash equilibria in circulant games.

Note that if $A_{i}$ is circulant then $a_{i j}=a_{j-i}$ and if $A_{i}$ is anti-circulant then $a_{i j}=a_{i+j}$ where the indices are to be read modulo $n$, e.g. $-1=n-1, n+1=1$, etc. In a circulant game, if player 1's payoff matrix is circulant then $\pi_{1}\left(s \mid s^{\prime}\right)=a_{s^{\prime}-s}^{1}$ and if player 1's payoff matrix is anti-circulant 
then $\pi_{1}\left(s \mid s^{\prime}\right)=a_{s+s^{\prime}}^{1}$. Similarly if player 2's payoff matrix is circulant then $\pi_{2}\left(s \mid s^{\prime}\right)=a_{s-s^{\prime}}^{2}$ and if player 2's payoff matrix is anti-circulant then $\pi_{2}\left(s \mid s^{\prime}\right)=a_{s+s^{\prime}}^{2}$. Throughout the paper the sum and difference of two strategies (and the multiplication of a strategy with an integer) in a circulant game is to be read modulo $n$.

In a circulant game the entries in the first row of player 1's payoff matrix (weakly) decrease when moving from left to right with $a_{0}^{1}$ being the unique maximum payoff. The entries in the first row of player 2's payoff matrix (weakly) decrease either when moving from the largest payoff to the right, or when moving from the largest payoff to the left. The shift $k$ is determined by the position of the unique maximum payoff in the first row of player 2's payoff matrix. A shift of $k=n$ corresponds to $a_{0}^{2}$ being player 2's largest payoff. A shift of $k=0$ is of course possible but for notational convenience is formally represented by a shift of $k=n$.

Since in a circulant game the sum of the payoffs in each row and each column is constant, if one player plays the completely uniformly mixed strategy, then all of the other player's pure strategies yield the same payoff. An immediate consequence of this is the following.

Lemma 2.1. Let $\Gamma_{n}$ be a circulant game. Then $\sigma^{*}=\left(\sigma_{1}^{*}, \sigma_{2}^{*}\right)$ where $\sigma_{i}^{*}(s)=1 / n$ for all $s \in S^{n}$, $i=1,2$, is a Nash equilibrium of $\Gamma_{n}$.

We can classify circulant games according to whether the players' payoff matrices "rotate" in the same or in opposite directions.

Definition 3. A circulant game is iso-circulant if the players' payoff matrices are either both circulant or both anti-circulant matrices. It is counter-circulant if one player's payoff matrix is circulant and the other player's payoff matrix is anti-circulant.

For $n=2$ every iso-circulant game is also counter-circulant and vice versa, as any circulant $2 \times 2$ matrix is also anti-circulant. For $n \geq 3$, however, the class of iso-circulant games is disjoint from the class of counter-circulant games. Iso-circulant games with shift $k=n$ capture the class of (weakly ordered) circulant coordination games.

Example 1 (Matching Pennies).

The game given by

$$
A_{1}=\left(\begin{array}{rr}
1 & -1 \\
-1 & 1
\end{array}\right), \quad A_{2}=\left(\begin{array}{rr}
-1 & 1 \\
1 & -1
\end{array}\right)
$$

is the well-known Matching Pennies game. Both players' payoff matrices are circulant (and anti-circulant) and for player 2, $a_{n-1}^{2}=a_{1}^{2}=1$ is the largest payoff. Hence, it is an isocirculant (and also a counter-circulant) game with shift $k=1$. [(1/2,1/2), (1/2,1/2)] is a Nash equilibrium of this game. As we will show later it is the unique one.

Example 2 (Rock-Paper-Scissors).

The game given by

$$
A_{1}=\left(\begin{array}{lll}
3 & 2 & 1 \\
2 & 1 & 3 \\
1 & 3 & 2
\end{array}\right), \quad A_{2}=\left(\begin{array}{lll}
1 & 2 & 3 \\
2 & 3 & 1 \\
3 & 1 & 2
\end{array}\right) .
$$

is Rock-Paper-Scissors. Strategies are labeled such that for player 1, strategy 0 is 'Rock', strategy 1 is 'Scissors', and strategy 2 is 'Paper' and for player 2, strategy 0 is 'Scissors', strategy 1 is 'Rock', and strategy 2 is 'Paper'. Both players' payoff matrices are anti-circulant and for player $2, a_{n-1}^{2}=a_{2}^{2}=3$ is the largest payoff. This is an iso-circulant game with shift $k=1$. $[(1 / 3,1 / 3,1 / 3),(1 / 3,1 / 3,1 / 3)]$ is a Nash equilibrium of this game. As we will see later it is the unique one. 
Example 3 ( $4 \times 4$ Coordination Game).

The game given by

$$
A_{1}=\left(\begin{array}{llll}
5 & 4 & 3 & 2 \\
2 & 5 & 4 & 3 \\
3 & 2 & 5 & 4 \\
4 & 3 & 2 & 5
\end{array}\right), \quad A_{2}=\left(\begin{array}{llll}
5 & 4 & 3 & 2 \\
2 & 5 & 4 & 3 \\
3 & 2 & 5 & 4 \\
4 & 3 & 2 & 5
\end{array}\right)
$$

is an example of an iso-circulant game with shift $k=4$ as both players' payoff matrices are circulant and for player 2, $a_{n-4}^{2}=a_{0}^{2}=5$ is the largest payoff. The uniform probability distribution over all pure strategies, $[(1 / 4,1 / 4,1 / 4,1 / 4),(1 / 4,1 / 4,1 / 4,1 / 4)]$, constitutes a Nash equilibrium. It is, however, not the only one. As we will see later, our results immediately imply that this game has 15 Nash equilibria.

The following two games are examples of counter-circulant games. In both games player 1's payoff matrix is anti-circulant and player 2's payoff matrix is circulant.

\section{Example 4.}

$$
A_{1}=\left(\begin{array}{llll}
4 & 3 & 2 & 1 \\
3 & 2 & 1 & 4 \\
2 & 1 & 4 & 3 \\
1 & 4 & 3 & 2
\end{array}\right), \quad A_{2}=\left(\begin{array}{llll}
1 & 4 & 3 & 2 \\
2 & 1 & 4 & 3 \\
3 & 2 & 1 & 4 \\
4 & 3 & 2 & 1
\end{array}\right)
$$

This is a counter-circulant game with shift $k=3$ as for player $2, a_{n-3}^{2}=a_{1}^{2}=4$ is the largest payoff. The uniform probability distribution over all pure strategies $[(1 / 4,1 / 4,1 / 4,1 / 4),(1 / 4,1 / 4,1 / 4,1 / 4)$ is a Nash equilibrium of this game. As we will see later this game has 3 Nash equilibria.

\section{Example 5.}

$$
A_{1}=\left(\begin{array}{lllll}
5 & 4 & 3 & 2 & 1 \\
4 & 3 & 2 & 1 & 5 \\
3 & 2 & 1 & 5 & 4 \\
2 & 1 & 5 & 4 & 3 \\
1 & 5 & 4 & 3 & 2
\end{array}\right), \quad A_{2}=\left(\begin{array}{lllll}
3 & 2 & 1 & 5 & 4 \\
4 & 3 & 2 & 1 & 5 \\
5 & 4 & 3 & 2 & 1 \\
1 & 5 & 4 & 3 & 2 \\
2 & 1 & 5 & 4 & 3
\end{array}\right)
$$

This is a counter-circulant game with shift $k=2$ as for player $2, a_{n-2}^{2}=a_{3}^{2}=5$ is the largest payoff. The uniform probability distribution over all pure strategies $[(1 / 5,1 / 5,1 / 5,1 / 5,1 / 5),(1 / 5,1 / 5,1 / 5$, is a Nash equilibrium of this game. As we will see later this game has 7 Nash equilibria.

\section{Main Results}

In this section we present the main results on the number and the structure of Nash equilibria in circulant games. We start by presenting some preliminary lemmata that we require to state the main results. All proofs are relegated to the appendix.

\subsection{Preliminaries}

Lemma 3.1. Let $\Gamma_{n}$ be a circulant game with shift $k$ in which player 1's payoff matrix is anti-circulant and let $d=\operatorname{gcd}(k, n)$.

(i) If $\Gamma_{n}$ is iso-circulant, then in any Nash equilibrium $\left(\sigma_{1}, \sigma_{2}\right)$, for all $s \in S^{n}, \sigma_{i}(s)=0$ if and only if $\sigma_{i}(s+k m)=0$ for all $m=0, \ldots, \frac{n}{d}-1, i=1,2$. 
(ii) If $\Gamma_{n}$ is counter-circulant, then in any Nash equilibrium $\left(\sigma_{1}, \sigma_{2}\right)$, for all $s \in S^{n}, \sigma_{1}(s)=0$ if and only if $\sigma_{1}(-s+k)=0$ and $\sigma_{2}(s)=0$ if and only if $\sigma_{2}(-s-k)=0$

Given an iso-circulant game $\Gamma_{n}$, we can define an equivalence relation $\sim$ on the set $S^{n}$ by $s \sim s^{\prime}$ if and only if $s=s^{\prime}+m k$ for some $0 \leq m \leq \frac{n}{d}-1$, where $d=\operatorname{gcd}(n, k)$. Denote the equivalence class of $s \in S^{n}$ by $I(s)$. Note that, $s^{\prime}+m_{1} k \neq s^{\prime}+m_{2} k$ for all $0 \leq m_{1}<m_{2} \leq \frac{n}{d}-1$. Hence $I(s)=\left\{s+m k \mid 0 \leq m \leq \frac{n}{d}-1\right\}$ contains $n / d$ elements and there are $d$ different equivalence classes. Let $I\left(S^{n}\right)=\left\{I(s) \mid s \in S^{n}\right\}$ be the set of equivalence classes. Suppose player 1's payoff matrix is anti-circulant. By Lemma 3.1(i) two strategies are equivalent if and only if in any Nash equilibrium either both are simultaneously played with positive probability or both are simultaneously played with zero probability.

For a counter-circulant game let $C_{1}(s)=\{s,-s+k\}$ and $C_{2}(s)=\{s,-s-k\}$ for all $s \in S^{n}$. Note that any class $C_{1}(s)$ contains at least one and at most two elements. It contains one element if $-s+k \equiv s \bmod n$ and two elements if $-s+k \not \equiv s \bmod n$. The former occurs if and only if either $2 s=k$ or $2 s=n+k$. Thus there is a singleton class if and only if either $\frac{k}{2} \in S^{n}$ or $\frac{(n+k)}{2} \in S^{n}$, i.e. if either $k$ or $(n+k)$ is an even number. In particular there can be at most two singleton classes. Similarly, any class $C_{2}(s)$ contains one element if $-s-k \equiv s \bmod n$ and two elements if $-s-k \not \equiv s \bmod n$. The former occurs if and only if either $2 s=n-k$ or $2 s=2 n-k$. Thus there is a singleton class if and only if either $n-k$ or $2 n-k$ is an even number, which holds if and only if either $k$ or $(n+k)$ is an even number, i.e. if and only if $\frac{k}{2} \in S^{n}$ or $\frac{(n+k)}{2} \in S^{n}$. We define $C_{i}\left(S^{n}\right):=\left\{C_{i}(s) \mid s \in S^{n}\right\}, i=1,2$. Suppose player 1's payoff matrix is anti-circulant. Then, by Lemma 3.1(ii), $s^{\prime} \in C_{i}(s)$ if and only if in any Nash equilibrium either both $s$ and $s^{\prime}$ are simultaneously played with positive probability or both are simultaneously played with zero probability. For an iso-circulant game, the set $I\left(S^{n}\right)$ is a partition of $S^{n}$ by construction. It can be shown (Lemma B.3 in the appendix) that this is also true in the case of counter-circulant games and $C_{1}\left(S^{n}\right)$ and $C_{2}\left(S^{n}\right)$, respectively.

The following lemma covers the connection between the support of a strategy of player $i$ and the best response of player $-i$ against that strategy.

Lemma 3.2. Let $\Gamma_{n}$ be a circulant game in which player 1's payoff matrix is anti-circulant.

(i) If $\Gamma_{n}$ is iso-circulant then if $\sigma_{i} \in \Sigma_{i}$ and $I(s) \in I\left(S^{n}\right)$ are such that $\operatorname{supp}\left(\sigma_{i}\right) \cap I(s)=\emptyset$ then $B R_{-i}\left(\sigma_{i}\right) \cap I(-s)=\emptyset$.

(ii) If $\Gamma_{n}$ is counter-circulant then if $\operatorname{supp}\left(\sigma_{-i}\right) \cap C_{-i}(s)=\emptyset$ for $C_{-i}(s) \in C_{-i}\left(S^{n}\right)$ then $B R_{i}\left(\sigma_{-i}\right) \cap C_{i}(-s)=\emptyset$.

\subsection{The Number of Nash Equilibria}

Theorem 1. Let $\Gamma_{n}$ be an iso-circulant game with shift $k$ and let $d=\operatorname{gcd}(k, n)$ denote the greatest common divisor of $k$ and $n$. Then $\Gamma_{n}$ has $2^{d}-1$ Nash equilibria.

Since by definition $k \leq n$, necessarily $\operatorname{gcd}(k, n) \leq n$. It follows that an iso-circulant game can have at most $2^{n}-1$ Nash equilibria. Further, an iso-circulant game has a unique Nash equilibrium if and only if $\operatorname{gcd}(k, n)=1$. Together with Lemma 2.1 , this implies that if $\operatorname{gcd}(k, n)=1$ then the unique Nash equilibrium is the one where both players place equal probability on each pure strategy. Some immediate consequences of these results are the following.

Matching Pennies (Example 1) is an iso-circulant game with shift $k=1$. Hence, $[(1 / 2,1 / 2),(1 / 2,1 / 2)]$ is the unique Nash equilibrium. Rock-Paper-Scissors (Example 2) is an iso-circulant game with shift $k=1$. Hence, the unique Nash equilibrium is $[(1 / 3,1 / 3,1 / 3),(1 / 3,1 / 3,1 / 3)]$. 
Proposition 3. Let $\Gamma_{n}$ be an iso-circulant game with shift $k . \Gamma_{n}$ has $n$ pure strategy Nash equilibria if and only if $k=n$. Further, $\Gamma_{n}$ has no pure strategy Nash equilibrium if and only if $k \neq n$.

By the last proposition an iso-circulant game $\Gamma_{n}$ has either 0 or $n$ pure strategy Nash equilibria. The $4 \times 4$ coordination game in Example 3 is an iso-circulant game with shift $k=4$. As $\operatorname{gcd}(4,4)=4$, by Theorem 1 , this game has $2^{4}-1=15$ Nash equilibria. By Proposition 3 four of these are in pure strategies.

Theorem 2. Let $\Gamma_{n}$ be a counter-circulant game with shift $k$.

(i) If $n$ is odd, then $\Gamma_{n}$ has exactly $2^{\frac{n+1}{2}}-1$ Nash equilibria.

(ii) If both $n$ and $k$ are even, then $\Gamma_{n}$ has exactly $2^{\frac{n}{2}+1}-1$ Nash equilibria.

(iii) If $n$ is even and $k$ is odd, then $\Gamma_{n}$ has exactly $2^{\frac{n}{2}}-1$ Nash equilibria.

It follows that a counter-circulant game can have at most $2^{\frac{n}{2}+1}-1$ Nash equilibria. Further, a counter-circulant game has a unique Nash equilibrium if and only if $n=2$ and $k=1$. Example 4 is a counter-circulant game with shift $k=3$. As $n$ is even and $k$ is odd, by Theorem 2 (iii) the game has $2^{2}-1=3$ Nash equilibria. Example 5 is a counter-circulant game with shift $k=2$. As $n$ is odd, by Theorem 2(i) the game has $2^{3}-1=7$ Nash equilibria.

Proposition 4. Let $\Gamma_{n}$ be a counter-circulant game with shift $k$.

(i) $\Gamma_{n}$ has exactly one pure strategy Nash equilibrium if and only if $n$ is odd.

(ii) $\Gamma_{n}$ has exactly two pure strategy Nash equilibria if and only if both $n$ and $k$ are even.

(iii) $\Gamma_{n}$ has no pure strategy Nash equilibrium if and only if $n$ is even and $k$ is odd.

In Example $4 n$ is even and $k$ is odd, hence by Proposition 4(iii) none of its three Nash equilibria are in pure strategies. In Example $5 n$ is odd, hence by Proposition 4(i) one of its seven Nash equilibria is in pure strategies.

It follows from (i) and (ii) in Proposition 4 that the class of counter-circulant games with even shift is a class of games for which a pure strategy Nash equilibrium always exists.

\subsection{The Structure of Nash Equilibria}

The next lemma shows that only specific subsets of $S^{n}$ can arise as the support of a Nash equilibrium strategy of player 1.

Lemma 3.3. Let $\Gamma_{n}$ be a circulant game in which player 1's payoff matrix is anti-circulant.

(i) If $\Gamma_{n}$ is iso-circulant then for any union $U=\bigcup_{j=1}^{m} I\left(s^{j}\right)$ of elements of $I\left(S^{n}\right)$ there is a unique Nash equilibrium $\left(\sigma_{1}, \sigma_{2}\right)$ such that $\operatorname{supp}\left(\sigma_{1}\right)=U$. Further, for any Nash Equilibrium $\left(\sigma_{1}, \sigma_{2}\right)$ there is a union $U=\bigcup_{j=1}^{m} I\left(s^{j}\right)$ of elements of $I\left(S^{n}\right)$ such that $\operatorname{supp}\left(\sigma_{1}\right)=U$.

(ii) If $\Gamma_{n}$ is counter-circulant then for any union $U=\bigcup_{j=1}^{m} C_{1}\left(s^{j}\right)$ of elements of $C_{1}\left(S^{n}\right)$ there is a unique Nash equilibrium $\left(\sigma_{1}, \sigma_{2}\right)$ such that $\operatorname{supp}\left(\sigma_{1}\right)=U$. Further, for any Nash Equilibrium $\left(\sigma_{1}, \sigma_{2}\right)$ there is a union $U=\bigcup_{j=1}^{m} C_{1}\left(s^{j}\right)$ of elements of $C_{1}\left(S^{n}\right)$ such that $\operatorname{supp}\left(\sigma_{1}\right)=U$. 
By Lemma 3.3, there exists a straightforward way to characterize the support of all Nash equilibrium strategies for a given circulant game. Moreover, once we know what to look for the weights of the strategies in the support can be easily derived.

Consider first the case of an iso-circulant game with $n$ and $k$, and let $d=\operatorname{gcd}(n, k)$. We can transform the game so that player 1's payoff matrix is anti-circulant (see Lemma A.1(i) in the appendix). Recall that by Lemma 3.1(i) the circulant structure of the payoff matrices allows us to define an equivalence relation on the set of pure strategies $S^{n}$ for each player. For a pure strategy $s \in S^{n}$, the corresponding equivalence class $I(s)=\left\{s+m k \mid 0 \leq m \leq \frac{n}{d}-1\right\}$ contains $n / d$ elements and there are $d$ different equivalence classes. In any Nash equilibrium all strategies within one equivalence class are either played with strictly positive or with zero probability. It follows from Lemma 3.3(i) that in any Nash equilibrium the support of either player's strategy is the union of classes in $I\left(S^{n}\right)=\left\{I(s) \mid s \in S^{n}\right\}$ and further that for any such union of classes in $I\left(S^{n}\right)$ there is a unique Nash equilibrium in which player 1's strategy has this union as its support. Further, if the mixed strategy profile $\left(\sigma_{1}, \sigma_{2}\right)$ is a Nash equilibrium with $\operatorname{supp}\left(\sigma_{1}\right)=\bigcup_{j=1}^{m} I\left(s^{j}\right)$ for some strategies $s^{1}, \ldots, s^{m} \in S^{n}$ then by Lemma 3.2(i) it follows that $\operatorname{supp}\left(\sigma_{2}\right)=\bigcup_{j=1}^{m} I\left(-s^{j}\right)$. The actual probabilities for each pure strategy of course depend on the actual payoffs, however, the structure of the supports is the same for all iso-circulant games with the same shift and the same number of pure strategies.

Let us revisit the $4 \times 4$ Coordination game from Example 3. We can transform this game so that both payoff matrices are anti-circulant (see Table 1 and Lemma A.1(i) in the appendix). In this game $n=k=d=4$ and hence there are four (singleton) classes: $I(0)=\{0\}, I(1)=\{1\}$, $I(2)=\{2\}$, and $I(3)=\{3\}$. Each class is part of a (pure strategy) Nash equilibrium in which $\operatorname{supp}\left(\sigma_{1}\right)=I(s)$ and $\operatorname{supp}\left(\sigma_{2}\right)=I(-s)$, and there are four such combinations. For instance, in one Nash equilibrium player 1 plays the strategy $s=1$, i.e. chooses support $I(1)$ and player 2 plays $s=3$, chooses support $I(-1)=I(3)$. Analogously, the three remaining pure strategy Nash equilibria are given by the profiles $(0,0),(2,2)$, and $(3,1)$. Further, each union of two classes is part of a (mixed strategy) Nash equilibrium in which $\operatorname{supp}\left(\sigma_{1}\right)=I\left(s^{1}\right) \cup I\left(s^{2}\right)$ and $\operatorname{supp}\left(\sigma_{2}\right)=I\left(-s^{1}\right) \cup I\left(-s^{2}\right)$. There are six such combinations, e.g., in one Nash equilibrium player 1 puts positive probability only on $I(0)$ and $I(1)$ and player 2 puts positive probability on $I(-0) \cup I(-1)=I(0) \cup I(3)$. The probabilities are easily derived from the corresponding indifference conditions and the Nash equilibrium strategy profile is $[(1 / 4,3 / 4,0,0),(3 / 4,0,0,1 / 4)]$. Similarly, there are four Nash equilibria in which the support of player 1's (and player 2's) strategy is the union of three classes, e.g., $[(1 / 4,1 / 4,1 / 2,0),(1 / 2,0,1 / 4,1 / 4)]$. Finally, there is one Nash equilibrium where player 1's (and player 2's) strategy put positive probability on all four equivalence classes, i.e. plays a completely mixed strategy: $[(1 / 4,1 / 4,1 / 4,1 / 4)$, $(1 / 4,1 / 4,1 / 4,1 / 4)] .^{3}$

Consider now the case of a counter-circulant game with given $n$ and $k$. We can transform this game so that player 1's payoff matrix is anti-circulant (see Lemma A.1(ii) in the appendix). Recall that by Lemma 3.1(ii) we can define an equivalence relation on set of pure strategies for each player. For all $s \in S$ let $C_{1}(s)=\{s,-s+k\}$ denote the corresponding equivalence class of player 1 and $C_{2}(s)=\{s,-s-k\}$ the one of player 2. Note that any class $C_{1}(s), C_{2}(s)$ contains at least one and at most two elements. It follows from Lemma 3.3(ii) that in any Nash equilibrium the support of player 1's strategy is a union of classes in $C_{1}\left(S^{n}\right)=\left\{C_{1}(s) \mid s \in S^{n}\right\}$ and that for any union of classes in $C_{1}\left(S^{n}\right)$ there is a Nash equilibrium in which the support of player 1's strategy has this union as its support. Further, if $\left(\sigma_{1}, \sigma_{2}\right)$ is a Nash equilibrium with $\operatorname{supp}\left(\sigma_{1}\right)=\bigcup_{j=1}^{m} C_{1}\left(s^{j}\right)$ for some strategies $s^{1}, \ldots, s^{m} \in S^{n}$ then by Lemma 3.2(ii) it follows that $\operatorname{supp}\left(\sigma_{2}\right)=\bigcup_{j=1}^{m} C_{2}\left(-s^{j}\right)$.

Let us revisit the game in Example 4. Here, $n=4$ and $k=3$. There are two classes for

\footnotetext{
${ }^{3}$ Table 1 in the appendix contains the remaining Nash equilibrium profiles.
} 
player 1: $C_{1}(0)=C_{1}(3)=\{0,3\}$ and $C_{1}(1)=C_{1}(2)=\{1,2\}$. Correspondingly there are two classes for player 2: $C_{2}(0)=C_{2}(1)=\{0,1\}$ and $C_{2}(2)=C_{2}(3)=\{2,3\}$. There are two Nash equilibria in which the support of player 1's (and player 2's) strategy consists of a single class, e.g. $[(1 / 4,0,0,3 / 4),(1 / 4,3 / 4,0,0)]$. Further there is one equilibrium in which both players play the completely mixed strategy $\left[(1 / 4,1 / 4,1 / 4,1 / 4){ }^{4}\right.$

\section{Generalizations}

By our definition there are games that are not circulant games, but can be transformed into one by a simple relabeling of strategies. We chose to exclude those games from our definition for ease of exposition. However, the results presented above also apply for these games.

It is not necessary to insist on each row containing the same entries. All our proofs go through if payoffs are transformed in a way that preserves the order of entries in each row and in each column of the payoff matrices.

Example 6. In the $3 \times 3$ game with payoff matrices

$$
A_{1}=\left(\begin{array}{lll}
3.1 & 1.9 & 0.8 \\
1.5 & 0.9 & 3.4 \\
0.5 & 3.2 & 2.1
\end{array}\right), \quad A_{2}=\left(\begin{array}{lll}
0.7 & 2.2 & 3.5 \\
1.8 & 2.6 & 0.1 \\
3.0 & 0.5 & 2.8
\end{array}\right)
$$

the order of payoffs in each row and in each column is the same as in Rock-Paper-Scissors (Example 2). The proof of Theorem 1 can easily be generalized to this case to show that this game has a unique Nash equilibrium. As the sum of payoffs in each row is not constant, however, the unique Nash equilibrium is not the strategy profile in which both players play the uniformly mixed strategies.

In this sense, our results on the number and the structure of Nash equilibria only depend on the order of payoffs in the rows and columns of the payoff matrices.

Our results further generalize to coordination games in which players obtain a strictly positive payoff if and only if they use the same strategy and a payoff of 0 otherwise i.e., so-called games of pure coordination. The resulting payoff matrices are of the form

$$
A_{1}=\left(\begin{array}{ccccc}
a_{0} & 0 & 0 & \cdots & 0 \\
0 & a_{1} & 0 & \cdots & 0 \\
0 & 0 & a_{2} & \cdots & 0 \\
\vdots & \vdots & \vdots & \ddots & \vdots \\
0 & 0 & 0 & \cdots & a_{n-1}
\end{array}\right), \quad A_{2}=\left(\begin{array}{ccccc}
a_{0} & 0 & 0 & \cdots & 0 \\
0 & a_{1} & 0 & \cdots & 0 \\
0 & 0 & a_{2} & \cdots & 0 \\
\vdots & \vdots & \vdots & \ddots & \vdots \\
0 & 0 & 0 & \cdots & a_{n-1}
\end{array}\right)
$$

Proving that such games have $2^{n}-1$ Nash equilibria works analogously to the proof of Theorem 1.

\section{Conclusion}

In this paper we introduce and investigate a class of two-player normal-form games we coin circulant games. Such games have a straightforward representation in form of circulant matrices.

\footnotetext{
${ }^{4}$ Table 2 in the appendix shows the Nash equilibria and the equivalence classes for the two counter-circulant games we introduced in Example 4 and 5.
} 
Each player's payoff matrix is fully characterized by a single row vector, which is rotated to obtain the rest of the matrix. All circulant games have a Nash equilibrium where players randomize between all pure strategies with equal probability (uniformly mixed Nash equilibrium), but might have many other pure and mixed Nash equilibria.

The circulant structure underlying the payoff matrices has interesting implications. First, the best response correspondences induce a partition on each players' set of pure strategies into equivalence classes. In any Nash Equilibrium all strategies within one class are either played with strictly positive or with zero probability. Second, there exists a simple one-to-one correspondence between the players' respective equivalence classes. If some player puts zero probability on one class, the other has one corresponding equivalence class he plays with zero probability. Finally, a single parameter $k$ fully determines the strategy classes and the relation between the players' classes. The parameter itself only depends on the position of the largest payoff in the first row of a player's payoff matrix. For a given circulant game, knowing $k$ and the number of pure strategies $n$ suffices to calculate the exact number of Nash equilibria and to describe the support of all Nash equilibrium strategies. As an immediate consequence of our main results we establish $2^{n}-1$ as the tight upper bound on the number of Nash equilibria in these games.

The class of circulant games contains a large variety of games with cyclical payoff structures including well-known games such as Matching Pennies, Rock-Paper-Scissors or subclasses of coordination and common interest games. We shed new light on the features these games have in common focusing on the circulant structure of their payoff matrices. For example, Matching Pennies is not only the two-strategy variant of Rock-Paper-Scissors. Beyond their common zero-sum properties the two games belong to the same sub-class of circulant games. Both are characterized by $k=1$ and the only Nash equilibrium is the uniformly mixed one. The common denominator that connects these games is the balanced payoff structure induced by the circulant matrices with a shift of $k=1$. Moreover, this reinterpretation is robust in the sense that only relative payoffs matter. We can write down many variants of Rock-Paper-Scissors, including asymmetric evaluations of wins or losses and variants that cannot be transformed into zero-sum games. Yet, the balanced structure is preserved and the best players can do is to randomize between all pure strategies with equal probability.

\section{Acknowledgments}

We would like to thank Carlos Alós-Ferrer, Tanja Artiga Gonzalez, Wolfgang Leininger, participants at the SAET13 conference in Paris, participants at the Jahrestagung des Vereins für Socialpolitik 2013 in Düsseldorf, and seminar participants in Cologne and Innsbruck for helpful comments and discussions. Johannes Kern gratefully acknowledges financial support from the German Research Foundation through research projects AL-1169/1-1 and AL-1169/1-2. Đura-Georg Granić also gratefully acknowledges financial support from the German Research Foundation (DFG) through research project AL-1169/2-1.

\section{References}

Alós-Ferrer, C., And C. Kuzmics (2013): "Hidden Symmetries and Focal Points," Journal of Economic Theory, 148(1), 226-258.

BAhel, E. (2012): "Rock-Paper-Scissors and Cycle-Based Games," Economics Letters, 115(3), 401-403. 
Bahel, E., And H. Haller (2013): "Cycles With Undistinguished Actions and Extended Rock-Paper-Scissors Games," Economics Letters, 120(3), 588-591.

Casajus, A. (2000): "Focal Points in Framed Strategic Forms," Games and Economic Behavior, 32(3), 263-291.

Davis, P. J. (1979): Circulant Matrices. Wiley, New York.

Diekmann, O., And S. A. van Gils (2009): "On the Cyclic Replicator Equation and the Dynamics of Semelparous Populations," SIAM Journal on Applied Dynamical Systems, 8(3), 1160-1189.

Duersch, P., J. Oechssler, And B. C. Schipper (2012): "Pure Strategy Equilibria in Symmetric Two-Player Zero-Sum Games," International Journal of Game Theory, 41(3), $553-564$.

Hofbauer, J., P. Schuster, K. Sigmund, And R. Wolff (1980): "Dynamical Systems Under Constant Organization II: Homogeneous Growth Functions of Degree $\mathrm{p}=2$," SIAM Journal on Applied Mathematics, 38(2), 282-304.

Hofbauer, J., And K. Sigmund (1998): Evolutionary Games and Population Dynamics. Cambridge University Press.

Janssen, M. C. (2001): "Rationalizing Focal Points," Theory and Decision, 50(3), 119-148.

KeIDing, H. (1998): "On the Structure of the Set of Nash Equilibria of Weakly Nondegenerate Bimatrix Games," Annals of Operations Research, 84, 231-238.

NAsh, J. F. (1951): "Non-Cooperative Games," Annals of Mathematics, 54(2), 286-295.

Quint, T., And M. Shubik (1997): "A Theorem on the Number of Nash Equilibria in a Bimatrix Game," International Journal of Game Theory, 26(3), 353-359.

Quint, T., And M. Shubik (2002): "A Bound on the Number of Nash Equilibria in a Coordination Game," Economics Letters, 77(3), 323-327.

Sandholm, W. (2010): Population Games and Evolutionary Dynamics, Economic Learning and Social Evolution. MIT Press.

Shapley, L. (1974): "A Note on the Lemke-Howson Algorithm," Mathematical Programming Study, 1, 175-189.

Sinervo, B., And C. M. Lively (1996): "The Rock-Paper-Scissors Game and the Evolution of Alternative Male Strategies," Nature, 380(6571), 240-243.

von Stengel, B. (1997): "New Lower Bounds for the Number of Equilibria in Bimatrix Games," Technical Report 264, Dept. of Computer Science, ETH Zürich.

(1999): "New Maximal Numbers of Equilibria in Bimatrix Games," Discrete \&6 Computational Geometry, 21(4), 557-568. 


\section{A Transformation of Games}

Lemma A.1. (i) Let $\Gamma_{n}$ be an iso-circulant game in which both players' payoff matrices are circulant. There is a permutation of row vectors that fixes the first row in both matrices and transforms both players' payoff matrices into anti-circulant matrices.

(ii) Let $\Gamma_{n}$ be a counter-circulant game in which player 1's payoff matrix is circulant. There is a permutation of row vectors that fixes that first row in both matrices and transforms player 1's payoff matrix into an anti-circulant matrix and player 2's matrix into a circulant matrix.

Proof. (i) A matrix $A$ is anti-circulant if and only if $A=P C$, where $C$ is a circulant matrix and

$$
P=\left(\begin{array}{cccccc}
1 & 0 & \cdots & 0 & 0 & 0 \\
0 & 0 & \cdots & 0 & 0 & 1 \\
0 & 0 & \cdots & 0 & 1 & 0 \\
0 & 0 & \cdots & 1 & 0 & 0 \\
\vdots & \vdots & & \vdots & \vdots & \vdots \\
0 & 1 & \cdots & 0 & 0 & 0
\end{array}\right)
$$

(Davis, 1979, p. 162, Corollary). The matrix $P$ switches rows $i$ and $n+1-i$ and fixes the first row. Using this result, we obtain that $P A_{1}$ and $P A_{2}$ are anti-circulant matrices since both $A_{1}$ and $A_{2}$ are circulant matrices.

(ii) Using the matrix $P$ defined as in (i), we obtain that $P A_{1}$ is anti-circulant (Davis, 1979, p. 162, Corollary). As $A_{2}$ is anti-circulant, $A_{2}=P C$ for some circulant matrix $C$ (Davis, 1979, p. 162, Corollary). Hence $P A_{2}=P(P C)$ and since $P=P^{-1}$ (Davis, 1979, p.28, equ. (2.4.22)), we obtain that $P A_{2}$ is a circulant matrix.

\section{B Proofs of Main Results}

We remind the reader that the sum and the difference of strategies in a circulant game as well as multiplications of integers with strategies are read modulo $n$. Central to the proofs of our main results is Proposition 5 below.

Proposition 5 identifies sufficient conditions under which the number of Nash equilibria of a finite two player normal-form game can be calculated by merely identifying one parameter of the game. Under the hypotheses of Proposition 5, each Nash equilibrium strategy of a player corresponds to one specific combination of elements of a partition of that player's strategy set. Moreover, for each possible combination of elements of the partition there exists exactly one corresponding Nash equilibrium strategy. The parameter necessary to determine the number of Nash equilibria is the cardinality of the partition.

The proof of Theorem 1 (Theorem 2) first establishes that iso-circulant (counter-circulant) games satisfy the hypotheses of Proposition 5. Once this is done, determining the cardinality of the partitions is merely a counting exercise.

Proposition 5. For the two-player normal-form game $\Gamma_{n}$ let $\bar{S}_{1}=\left\{[s]_{1} \mid s \in S^{n}\right\}$ and $\bar{S}_{2}=$ $\left\{[s]_{2} \mid s \in S^{n}\right\}$ be partitions of $S^{n}$ such that $\left|\bar{S}_{1}\right|=\left|\bar{S}_{2}\right|$. If $\Gamma_{n}, \bar{S}_{1}$, and $\bar{S}_{2}$ satisfy 
(a) for all Nash equilibria $\left(\sigma_{1}, \sigma_{2}\right)$, and all $s, s^{\prime} \in S^{n}$, if $s^{\prime} \in[s]_{i}$ then $\sigma_{i}(s)=0$ if and only if $\sigma_{i}\left(s^{\prime}\right)=0$,

(b) for all $\sigma_{i} \in \Sigma_{i}, i=1,2, \operatorname{supp}\left(\sigma_{i}\right) \cap[s]_{i}=\emptyset$ for $[s]_{i} \in \bar{S}_{i}$ implies $B R_{-i}\left(\sigma_{i}\right) \cap[-s]_{-i}=\emptyset$,

(c) for all $s \in S^{n}, \Gamma_{n}$ has a Nash equilibrium $\left(\sigma_{1}, \sigma_{2}\right)$ with $\operatorname{supp}\left(\sigma_{1}\right)=[s]_{1}$ and $\operatorname{supp}\left(\sigma_{2}\right)=$ $[-s]_{2}$,

then

(i) for any $M \subseteq \bar{S}_{1} \Gamma_{n}$ has a unique Nash equilibrium $\left(\sigma_{1}, \sigma_{2}\right)$ with $\operatorname{supp}\left(\sigma_{1}\right)=\bigcup_{[s]_{1} \in M}[s]_{1}$;

(ii) $\Gamma_{n}$ has exactly $2^{\left|\bar{S}_{1}\right|}-1$ Nash equilibria.

Proof. (i) Given $\emptyset \neq M \subseteq \bar{S}_{1}$ let $-M:=\left\{[-s]_{2} \mid[s]_{1} \in M\right\} \subseteq$ and let $\Gamma_{n}^{M}$ be the reduced game where player 1's set of strategies is $\bigcup_{[s]_{1} \in M}[s]_{1}$ and player 2's set of strategies is $\bigcup_{[s]_{1} \in M}[-s]_{2}$ (and the payoff functions are restricted accordingly).

Claim A: Let $M^{\prime} \subseteq M \subseteq \bar{S}_{1}$ be a nonempty subset of $\bar{S}_{1}$ and let $\left(\sigma_{1}^{M^{\prime}}, \sigma_{2}^{M^{\prime}}\right)$ be a completely mixed Nash equilibrium of $\Gamma_{n}^{M^{\prime}}$. Then $\left(\sigma_{1}^{M}, \sigma_{2}^{M}\right)$ defined by $\sigma_{1}^{M}(s)=\sigma_{1}^{M^{\prime}}(s)$ if $[s]_{1} \in M^{\prime}$ and $\sigma_{1}^{M}(s)=0$ otherwise, and $\sigma_{2}^{M}(s)=\sigma_{2}^{M^{\prime}}(s)$ if $[s]_{2} \in-M^{\prime}$ and $\sigma_{2}^{M}(s)=0$ otherwise is a Nash equilibrium in $\Gamma_{n}^{M}$.

Since $\left(\sigma_{1}^{M^{\prime}}, \sigma_{2}^{M^{\prime}}\right)$ is a completely mixed Nash equilibrium of $\Gamma_{n}^{M^{\prime}}$, all strategies in $\bigcup_{[s]_{1} \in M^{\prime}}[-s]_{2}$ yield the same payoff for player 2 against $\sigma_{1}^{M}$. By hypothesis $(\mathrm{b}), \operatorname{since} \operatorname{supp}\left(\sigma_{1}^{M}\right)=\bigcup_{[s] \in M^{\prime}}[s]$, no strategy outside $\bigcup_{[s]_{1} \in M^{\prime}}[-s]_{2}$ can be a best response for player 2 against $\sigma_{1}^{M}$. Analogously all strategies in $\bigcup_{[s]_{1} \in M^{\prime}}[s]_{1}$ yield the same payoff for player 1 against $\sigma_{2}^{M}$, and since $\operatorname{supp}\left(\sigma_{2}^{M}\right)=-\bigcup_{[s]_{1} \in M^{\prime}}[-s]_{2}$, no strategy outside $\bigcup_{[s]_{1} \in M^{\prime}}[s]_{1}$ is a best response for player 1 against $\sigma_{2}^{M}$. Hence, by Proposition $1,\left(\sigma_{1}^{M}, \sigma_{2}^{M}\right)$ is a Nash equilibrium in $\Gamma_{n}^{M}$. This proves the claim.

Claim B: For any $\emptyset \neq M \subseteq \bar{S}_{1}$, the reduced game $\Gamma_{n}^{M}$ has exactly one completely mixed Nash equilibrium.

Let $\emptyset \neq M \subseteq \bar{S}_{1}$ be such that $|M|=m$. We will prove the claim by induction over $m$. Note first, that by hypothesis (b), in any Nash equilibrium $\left(\sigma_{1}, \sigma_{2}\right)$ of $\Gamma_{n}^{M}, \operatorname{supp}\left(\sigma_{1}\right)$ is a union of elements of $M$.

For $m=1$, this follows by hypothesis (c). For $m>1$, by induction hypothesis we obtain that for all $\emptyset \neq M^{\prime} \subsetneq M$ the reduced game $\Gamma_{n}^{M^{\prime}}$ has a unique completely mixed Nash equilibrium. By Claim A, for every $\emptyset \neq M^{\prime} \subsetneq M$ there is a Nash equilibrium $\left(\sigma_{1}^{M}, \sigma_{2}^{M}\right)$ in $\Gamma_{n}^{M}$ with $\operatorname{supp}\left(\sigma_{1}^{M}\right)=$ $\bigcup_{[s] \in M^{\prime}}[s]$. As by Proposition 2(ii) for any $\emptyset \neq M^{\prime} \subsetneq M$ there can be at most one Nash equilibrium $\left(\sigma_{1}, \sigma_{2}\right)$ in $\Gamma_{n}^{M}$ with $\operatorname{supp}\left(\sigma_{1}\right)=M^{\prime}$ we obtain that there is exactly one such Nash equilibrium. This implies that $\Gamma_{n}^{M}$ has at least $2^{m}-2$ Nash equilibria.

Suppose there is no completely mixed Nash equilibrium in $\Gamma_{n}^{M}$. Then $\Gamma_{n}^{M}$ has exactly $2^{m}-2$ Nash equilibria. From hypotheses (a) and (b) it follows that $\Gamma_{n}$ is non-degenerate and hence that $\Gamma_{n}^{M}$ is non-degenerate. By Proposition 2(i) $\Gamma_{n}^{M}$ must have an odd number of Nash equilibria, which contradicts the fact that $2^{m}-2$ is even. Hence there is at least one completely mixed Nash equilibrium and again because $\Gamma_{n}^{M}$ is non-degenerate by Proposition 2(ii) there is exactly one. This proves the claim.

By Claim B, for $\emptyset \neq M \subseteq \bar{S}_{1}, \Gamma_{n}^{M}$ has exactly one completely mixed Nash equilibrium $\left(\sigma_{1}^{M}, \sigma_{2}^{M}\right)$. By Claim A, this induces a Nash equilibrium $\left(\sigma_{1}, \sigma_{2}\right)$ in $\Gamma_{n}$ with $\operatorname{supp}\left(\sigma_{1}\right)=$ $\bigcup_{[s]_{1} \in M}[s]_{1}$. Any Nash equilibrium $\left(\sigma_{1}^{\prime}, \sigma_{2}^{\prime}\right) \neq\left(\sigma_{1}, \sigma_{2}\right)$ with $\operatorname{supp}\left(\sigma_{1}^{\prime}\right)=\bigcup_{[s]_{1} \in M}[s]_{1}$ would induce 
a completely mixed Nash equilibrium in $\Gamma_{n}^{M}$ different from $\left(\sigma_{1}^{M}, \sigma_{2}^{M}\right)$, a contradiction. Hence $\Gamma_{n}$ has exactly one Nash equilibrium $\left(\sigma_{1}, \sigma_{2}\right)$ with $\operatorname{supp}\left(\sigma_{1}\right)=\bigcup_{[s]_{1} \in M}[s]_{1}$.

(ii) From (i) it follows that for any $\emptyset \neq M \subseteq \bar{S}_{1}$ there is a unique Nash equilibrium $\left(\sigma_{1}, \sigma_{2}\right)$ in $\Gamma_{n}$ such that $\operatorname{supp}\left(\sigma_{1}\right)=\bigcup_{[s]_{1} \in M}[s]_{1}$. Further, by hypothesis (a), for any Nash equilibrium $\left(\sigma_{1}, \sigma_{2}\right)$ of $\Gamma_{n}$ there is $\emptyset \neq M \subseteq \bar{S}_{1}$ such that $\operatorname{supp}\left(\sigma_{1}\right)=\bigcup_{[s]_{1} \in M}[s]_{1}$. As $\bar{S}_{1}$ has $2^{\left|\bar{S}_{1}\right|}-1$ nonempty subsets, $\Gamma_{n}$ has exactly $2^{\left|\bar{S}_{1}\right|}-1$ Nash equilibria.

The following lemma is required in the proofs of Lemmata 3.1 and 3.2.

Lemma B.1. Let $\Gamma_{n}$ be a circulant game with shift $k$ in which player 1's payoff matrix is anti-circulant.

(i) For all $\sigma_{2} \in \Sigma_{2}$ and all $s \in S^{n}$ if $\sigma_{2}(s)=0$ then $-s \notin B R_{1}\left(\sigma_{2}\right)$.

(ii) If $\Gamma_{n}$ is iso-circulant, then for all $\sigma_{1} \in \Sigma_{1}$ and all $s \in S^{n}$ if $\sigma_{1}(s)=0$ then $(-s-k) \notin$ $B R_{2}\left(\sigma_{1}\right)$.

(iii) If $\Gamma_{n}$ is counter-circulant, then for all $\sigma_{1} \in \Sigma_{1}$ and all $s \in S^{n}$ if $\sigma_{1}(s)=0$ then $(s-k) \notin$ $B R_{2}\left(\sigma_{1}\right)$.

Proof. (i) Let $\sigma_{2} \in \Sigma_{2}$ be such that $\sigma_{2}(s)=0$ for some $s \in S^{n}$. Since player 1's payoff matrix is anti-circulant $\pi_{1}\left(s \mid s^{\prime}\right)=a_{s+s^{\prime}}^{1}$. We will show that there exists a strategy for player 1 that yields a strictly higher payoff against $\sigma_{2}$ than strategy $-s$. Let $l:=\min \left\{s<l^{\prime} \leq s+n-1 \mid \sigma_{2}\left(l^{\prime}\right)>0\right\}$. Since $n>1$ the set $\left\{s<l^{\prime} \leq s+n-1 \mid \sigma_{2}\left(l^{\prime}\right)>0\right\}$ is non-empty and $l$ exists. By construction of $l, \sigma_{2}(s)=\cdots=\sigma_{2}(l-1)=0$. We claim that $\pi_{1}\left(-s \mid \sigma_{2}\right)<\pi_{1}\left(-l \mid \sigma_{2}\right)$. To see this, note that

$$
\pi_{1}\left(-s \mid \sigma_{2}\right)=\sum_{t=l}^{s+n-1} \sigma_{2}(t) a_{t-s}^{1}
$$

and

$$
\pi_{1}\left(-l \mid \sigma_{2}\right)=\sum_{t=l}^{s+n-1} \sigma_{2}(t) a_{t-l}^{1} .
$$

Comparing these payoffs for $t=l$ we obtain that $a_{t-l}^{1}=a_{0}^{1}>a_{t-s}^{1}=a_{l-s}^{1}$, where the strict inequality holds by part (ii) of Definition 2. Further, for $l<t \leq s+n-1$ we have $0 \leq t-l<t-s \leq n-1$ and hence that $a_{t-l}^{1} \geq a_{t-s}^{1}$ again by part (ii) of Definition 2 . Since by construction of $l, \sigma_{2}(l)>0$ we obtain $\pi_{1}\left(-s \mid \sigma_{2}\right)<\pi_{1}\left(-l \mid \sigma_{2}\right)$ which proves the claim. Hence $-s \notin B R_{1}\left(\sigma_{2}\right)$.

(ii) Let $\sigma_{1} \in \Sigma_{1}$ and $s \in S^{n}$ be such that $\sigma_{1}(s)=0$. Since player 2's payoff matrix is anticirculant, $\pi_{2}\left(s \mid s^{\prime}\right)=a_{s^{\prime}+s}^{2}$ for $s, s^{\prime} \in S$. Since $\Gamma_{n}$ is a circulant game, by part (iii) of Definition 2 either $a_{n-k}^{2}>a_{n-k+1}^{2} \geq \cdots \geq a_{n-1}^{2} \geq a_{0}^{2} \geq a_{1}^{2} \geq \cdots \geq a_{n-k-1}^{2}$ or $a_{n-k}^{2}>a_{n-k-1}^{2} \geq \cdots \geq a_{1}^{2} \geq$ $a_{0}^{2} \geq a_{n-1}^{2} \geq \cdots \geq a_{n-k+1}^{2}$. We will only prove the result for the former case as the proof for the latter works analogously .

Let $l:=\min \left\{s<l^{\prime} \leq s+n-1 \mid \sigma_{1}\left(l^{\prime}\right)>0\right\}$ which exists since $\left\{s<l^{\prime} \leq s+n-1 \mid \sigma_{1}\left(l^{\prime}\right)>\right.$ $0\} \neq \emptyset$. Then $\sigma_{1}(s)=\cdots=\sigma_{1}(l-1)=0$. We claim that $\pi_{2}\left(-s-k \mid \sigma_{1}\right)<\pi_{2}\left(-l-k \mid \sigma_{1}\right)$. To see this, note that 


$$
\pi_{2}\left(-s-k \mid \sigma_{2}\right)=\sum_{t=l}^{s+n-1} \sigma_{1}(t) a_{t-s-k}^{2}
$$

and

$$
\pi_{2}\left(-l-k \mid \sigma_{2}\right)=\sum_{t=l}^{s+n-1} \sigma_{1}(t) a_{t-l-k}^{2} .
$$

For $t=l$ we have $a_{t-l-k}^{2}=a_{n-k}^{2}>a_{t-s-k}^{2}=a_{l-s-k}^{2}$, where the strict inequality holds by part (iii) of Definition 2. Further, for $l<t \leq s+n-1$ we have $a_{t-l-k}^{2} \geq a_{t-s-k}^{2}$ by part (iii) of Definition 2 since $t-l-k<t-s-k,-k \leq t-l-k<n-k-1$, and $-k<t-s-k \leq n-k-1$. Since by construction of $l, \sigma_{1}(l)>0$ we obtain that $\pi_{2}\left(-s-k \mid \sigma_{1}\right)<\pi_{2}\left(-l-k \mid \sigma_{1}\right)$ which proves the claim. Hence $(-s-k) \notin B R_{2}\left(\sigma_{1}\right)$.

(iii) Let $\sigma_{1} \in \Sigma_{1}$ and $s \in S^{n}$ be such that $\sigma_{1}(s)=0$. Since player 2's payoff matrix is circulant, $\pi_{2}\left(s \mid s^{\prime}\right)=a_{s-s^{\prime}}^{2}$ for $s, s^{\prime} \in S$. Since $\Gamma_{n}$ is a circulant game, by definition either $a_{n-k}^{2}>a_{n-k+1}^{2} \geq \cdots \geq a_{n-1}^{2} \geq a_{0}^{2} \geq a_{1}^{2} \geq \cdots \geq a_{n-k-1}^{2}$ or $a_{n-k}^{2}>a_{n-k-1}^{2} \geq \cdots \geq a_{1}^{2} \geq$ $a_{0}^{2} \geq a_{n-1}^{2} \geq \cdots \geq a_{n-k+1}^{2}$. We will only prove the result for the former case as the proof for the latter works analogously. Let $l:=\min \left\{s<l^{\prime} \leq s+n-1 \mid \sigma_{1}\left(l^{\prime}\right)>0\right\}$ which exists since $\left\{s<l^{\prime} \leq s+n-1 \mid \sigma_{1}\left(l^{\prime}\right)>0\right\} \neq \emptyset$. Then $\sigma_{1}(s)=\cdots=\sigma_{1}(l-1)=0$. We claim that $\pi_{2}\left(s-k \mid \sigma_{1}\right)<\pi_{2}\left(-l-k \mid \sigma_{1}\right)$. To see this, note that

$$
\pi_{2}\left(s-k \mid \sigma_{2}\right)=\sum_{t=l}^{s+n-1} \sigma_{1}(t) a_{s-k-t}^{2}
$$

and

$$
\pi_{2}\left(l-k \mid \sigma_{2}\right)=\sum_{t=l}^{s+n-1} \sigma_{1}(t) a_{l-k-t}^{2} .
$$

For $t=l$ we have $a_{l-k-t}^{2}=a_{n-k}^{2}>a_{s-k-t}^{2}=a_{s-k-l}^{2}$. Further, for $l<t \leq s+n-1$ we have $a_{l-k-t}^{2} \geq a_{s-k-t}^{2}$ by part (iii) of the definition of circulant game since $l-k-t>s-k-t$, $-k \geq l-k-t>-n-k+1$, and $-k>s-k-t \geq-n-k+1$. Since by construction of $l, \sigma_{1}(l)>0$ we obtain that $\pi_{2}\left(s-k \mid \sigma_{1}\right)<\pi_{2}\left(l-k \mid \sigma_{1}\right)$ which proves the claim. Hence $(s-k) \notin B R_{2}\left(\sigma_{1}\right)$.

Lemma B.1 allows us to rule out certain strategies as best responses for player $i$ if player $-i$ plays some strategy with zero probability in the case that player 1's payoff matrix is anticirculant. By (i) if player 2 plays a strategy $s$ with probability 0 then for player 1 strategy $-s$ cannot be a best response. Similarly, (ii) and (iii) state that if in an iso-circulant (countercirculant) game player 1 places probability 0 on strategy $s$ then $-s-k(s-k)$ cannot be a best response for player 2 .

We are now ready to prove Lemmata 3.1 and 3.2. It follows from Lemma 3.1(i) and Lemma 3.2(i) that iso-circulant games satisfy hypotheses (a) and (b) in Proposition 5. Analogously, Lemma 3.1(ii) and Lemma 3.2(ii) establish that counter-circulant games fulfill (a) and (b) in Proposition 5. 
Proof of Lemma 3.1. (i) The "if" part is trivial. To see the "only if" part let $\left(\sigma_{1}, \sigma_{2}\right)$ be a Nash equilibrium of $\Gamma_{n}$ and let $s \in S^{n}$ be such that $\sigma_{1}(s)=0$. By Lemma B.1(ii), $\sigma_{2}(-s-k)=0$ and consequently by Lemma B.1(i) $\sigma_{1}(s+k)=0$. Iterating this argument yields $\sigma_{1}(s+m k)=0$ for all $m=0, \ldots, \frac{n}{d}-1$. If $\sigma_{2}(s)=0$ the argument works analogously.

(ii) By Lemma B.1(i) and (iii) for any Nash equilibrium $\left(\sigma_{1}, \sigma_{2}\right)$ and any $s \in S^{n}$ we obtain

$$
\sigma_{1}(s)=0 \Rightarrow \sigma_{2}(s-k)=0 \Rightarrow \sigma_{1}(-s+k)=0
$$

and

$$
\sigma_{1}(-s+k)=0 \Rightarrow \sigma_{2}(-s)=0 \Rightarrow \sigma_{1}(s)=0 .
$$

Analogously, for player 2, we obtain

$$
\sigma_{2}(s)=0 \Rightarrow \sigma_{1}(-s)=0 \Rightarrow \sigma_{2}(-s-k)=0
$$

and

$$
\sigma_{2}(-s-k)=0 \Rightarrow \sigma_{1}(s+k)=0 \Rightarrow \sigma_{2}(s)=0 .
$$

Proof of Lemma 3.2. (i) First, let $s \in S^{n}$ be such that $\operatorname{supp}\left(\sigma_{1}\right) \cap I(s)=\emptyset$. By Lemma B.1(ii), $-s-(m+1) k \notin B R_{2}\left(\sigma_{1}\right)$ for all $0 \leq m \leq n / d-1$. As $\{-s-(m+1) k \mid 0 \leq m \leq n / d-1\}=I(-s)$ we obtain $B R_{2}\left(\sigma_{1}\right) \cap I(-s)=\emptyset$.

Next, let $s \in S^{n}$ be such that $\operatorname{supp}\left(\sigma_{2}\right) \cap I(s)=\emptyset$. By Lemma B.1(i), $-s-m k \notin B R_{1}\left(\sigma_{2}\right)$ for all $0 \leq m \leq n / d-1$. As $\{-s-m k \mid 0 \leq m \leq n / d-1\}=I(-s)$ we obtain $B R_{1}\left(\sigma_{2}\right) \cap I(-s)=\emptyset$.

(ii) If $\operatorname{supp}\left(\sigma_{-i}\right) \cap C_{-i}(s)=\emptyset$ for $C_{-i}(s) \in C_{-i}\left(S^{n}\right)$, then, since $C_{-i}(s)=\left\{s,-s+(-1)^{i-1} k\right\}$, by Lemma B.1(i) and (iii), $-s, s+(-1)^{i-1} k \notin B R_{i}\left(\sigma_{-i}\right)$. Hence $B R_{i}\left(\sigma_{-i}\right) \cap C_{i}(-s)=\emptyset$.

The following Lemma B.2 establishes that iso-circulant games fulfill hypothesis (c) in Proposition 5 and is used in the proofs of Theorem 1 and Proposition 3.

Lemma B.2. Let $\Gamma_{n}$ be an iso-circulant game in which both players' payoff matrices are anticirculant. For every $s \in S^{n}$, there is a Nash equilibrium $\left(\sigma_{1}, \sigma_{2}\right)$ such that $\operatorname{supp}\left(\sigma_{1}\right)=I(s)$ and $\operatorname{supp}\left(\sigma_{2}\right)=I(-s)$.

Proof. Given $\bar{s} \in S^{n}$, define $\sigma_{1}(s)=d / n$ for all $s \in I(\bar{s})$ and $\sigma_{2}(s)=d / n$ for all $s \in I(-\bar{s})$. By construction $\operatorname{supp}\left(\sigma_{1}\right)=I(\bar{s})$ and $\operatorname{supp}\left(\sigma_{2}\right)=I(-\bar{s})$. By Lemma 3.2(i), no strategy outside $I(\bar{s})$ can be a best response for player 1 against $\sigma_{2}$ and no strategy outside $I(-\bar{s})$ can be a best response for player 2 against $\sigma_{1}$. Further, $\pi_{1}\left(s \mid \sigma_{2}\right)=\sum_{m=0}^{n / d-1} \frac{d}{n} a_{s+\bar{s}+m k}=\pi_{1}\left(s^{\prime} \mid \sigma_{2}\right)$ for all $s, s^{\prime} \in I(\bar{s})$ and analogously $\pi_{2}\left(s \mid \sigma_{1}\right)=\pi_{2}\left(s^{\prime} \mid \sigma_{1}\right)$ for all $s, s^{\prime} \in I(-\bar{s})$. Proposition 1 yields that $\left(\sigma_{1}, \sigma_{2}\right)$ is a Nash equilibrium of $\Gamma_{n}$.

We are now ready to prove Theorem 1 and Proposition 3 . 
Proof of Theorem 1. If $\Gamma_{n}$ is an iso-circulant game in which both players' payoff matrices are anti-circulant then by Lemma 3.1(i), Lemma 3.2(i) and Lemma B.2, $\Gamma_{n}$ and $\bar{S}_{1}=\bar{S}_{2}=I\left(S^{n}\right)$ as defined in section 3.1 then satisfy the hypotheses of Proposition 5. As $\left|I\left(S^{n}\right)\right|=d$, it follows that $\Gamma_{n}$ has $2^{d}-1$ Nash equilibria. If $\Gamma_{n}$ is an iso-circulant game in which both players' payoff matrices are circulant, there is a permutation of row vectors that transforms both players' payoff matrices into anti-circulant matrices while fixing the first row in both matrices (Lemma A.1(i)). This permutation, which is essentially a relabeling of the players' strategies, does not affect the number of equilibria. Hence, the proof is complete.

Proof of Proposition 3. Note first that if both players' payoff matrices are circulant then by Lemma A.1(i) the game can be transformed into a different version of the same game in which both players' payoff matrices are anti-circulant by a permutation of row vectors. Since such a permutation does not affect the number of pure strategy Nash equilibria, we assume wlog that both players' payoff matrices are anti-circulant.

To see the "if" part suppose $k=n$. Then by construction, each class $I(s)$ is a singleton set and there are $n$ disjoint classes. Hence by Lemma B.2, $\Gamma_{n}$ has at least $n$ pure strategy Nash equilibria. By Lemma 3.1(i), in any pure strategy Nash equilibrium $\left(\sigma_{1}, \sigma_{2}\right), \operatorname{supp}\left(\sigma_{1}\right)=I(s)$ for some $s \in S$ and hence $\Gamma_{n}$ has exactly $n$ pure strategy Nash equilibria.

To prove the "only if" part let $\Gamma_{n}$ have $n$ pure strategy Nash equilibria and let $\left(s_{1}, s_{2}\right)$ be one of them. By Lemma 3.1(i), $I\left(s_{1}\right)$ must be a singleton set. By construction, $I\left(s_{1}\right)$ is a singleton set if and only if $k=n$.

This proves the first part of the theorem.

To see the second part, note that by construction of the classes $I(s)$ is a singleton set if and only if $k=n$ for any $s \in S$. Further by Lemma 3.1(i) and Lemma B.2, $\Gamma_{n}$ has a pure strategy Nash equilibrium if and only if there is a singleton equivalence class $I(s)$. Hence, $\Gamma_{n}$ has no pure strategy Nash equilibrium if and only if $k \neq n$.

Before we can turn to the proofs of Theorem 2 and Proposition 4 we require a couple more preliminary lemmata. One hypothesis in Proposition 5 requires the sets $\bar{S}_{1}$ and $\bar{S}_{2}$ to be partitions of the strategy set. While this is true by construction for $I\left(S^{n}\right)$ in the case of isocirculant games, the following Lemma B.3 shows that the $C_{1}\left(S^{n}\right)$ and $C_{2}\left(S^{n}\right)$ form a partition of $S^{n}$.

Lemma B.3. Let $\Gamma_{n}$ be a counter-circulant game. For $i=1,2$ the set $C_{i}\left(S^{n}\right)$ is a partition of $S^{n}$.

Proof. We will prove the result for $i=1$ as the proof for $i=2$ works analogously. Since $s \in C_{1}(s)$ for all $s \in S^{n}$, it follows that $\bigcup_{s \in S^{n}} C_{1}(s)=S^{n}$. If there is $\bar{s} \in C_{1}(s) \cap C_{1}\left(s^{\prime}\right)$ for some $s, s^{\prime} \in S^{n}$, then then since $\bar{s} \in C_{1}(s)$ either $\bar{s}=s$ or $\bar{s}=-s+k$. If $\bar{s}=s$ then $C_{i}(s)=C_{i}(\bar{s})$. If $\bar{s}=-s+k$ then $-\bar{s}+k=s-k+k=s$. In any case it follows that $C_{1}(\bar{s})=C_{1}(s)$. Using the same argument one obtains $C_{1}(\bar{s})=C_{1}\left(s^{\prime}\right)$ and hence that $C_{1}(s)=C_{1}\left(s^{\prime}\right)$. 
The following Lemma B.4 establishes that counter-circulant games fulfill property (c) in Proposition 5.

Lemma B.4. Let $\Gamma_{n}$ be a counter-circulant game in which player 1's payoff matrix is anticirculant and let $\sigma=\left(\sigma_{1}, \sigma_{2}\right) \in \Sigma_{1} \times \Sigma_{2}$.

(i) $C_{i}(s)$ is a singleton set if and only if $C_{-i}(-s)$ is a singleton set.

(ii) For every $s \in S^{n}$, there is a Nash equilibrium $\left(\sigma_{1}, \sigma_{2}\right)$ such that $\operatorname{supp}\left(\sigma_{1}\right)=C_{1}(s)$ and $\operatorname{supp}\left(\sigma_{2}\right)=C_{2}(-s)$.

Proof. (i) Suppose that $C_{i}(s)$ is a singleton. By construction, $s \equiv-s+(-1)^{i-1} k$ mod $n$ which is equivalent to $-s \equiv s+(-1)^{i} k \bmod n$. This holds if and only if $C_{-i}(-s)$ is a singleton.

(ii) Note that this follows from (i) and Lemma 3.2(ii) if $C_{1}(s)$ is a singleton set. Hence, suppose that $C_{1}(s)=\{s,-s+k\}$ contains two elements. Then, by (i), $C_{2}(-s)=\{-s, s-k\}$ contains two elements and neither $2 s=k$ nor $2 s=n+k$. Choose $\sigma_{1}(s)$ as the solution to $x a_{-2 s}^{2}+(1-x) a_{-k}^{2}=x a_{-k}^{2}+(1-x) a_{2 s-2 k}^{2}$, i.e.

$$
\sigma_{1}^{s}(s)=\frac{a_{2 s-2 k}^{2}-a_{n-k}^{2}}{a_{2 s-2 k}^{2}-a_{n-k}^{2}+a_{n-2 s}^{2}-a_{n-k}^{2}} .
$$

By definition $a_{n-k}^{2}$ is player 2's largest payoff implying that $a_{2 s-2 k}^{2}-a_{n-k}^{2}<0$ since $2 s \neq n+k$ and that $a_{n-2 s}^{2}-a_{n-k}^{2}<0$ since $2 s \neq k$. Hence $\left.\sigma_{1}(s) \in\right] 0,1[$.

Choose $\sigma_{2}^{s}(-s)$ as the solution to $x a_{0}^{1}+(1-x) a_{2 s-k}^{1}=x a_{-2 s+k}^{1}+(1-x) a_{0}^{1}$, i.e.

$$
\sigma_{2}^{s}(-s)=\frac{a_{0}^{1}-a_{2 s-k}^{1}}{a_{0}^{1}-a_{2 s-k}^{1}+a_{0}^{1}-a_{-2 s+k}^{1}} .
$$

By definition $a_{0}^{1}$ is player 1's largest payoff. Hence as $2 s \neq k a_{0}^{1}-a_{2 s-k}^{1}>0$ and $a_{0}^{1}-$ $a_{-2 s+k}^{1}>0$ implying that $\left.\sigma_{2}(-s) \in\right] 0,1\left[\right.$. By Lemma 3.2(ii) and Proposition 1, $\left(\sigma_{1}, \sigma_{2}\right)$ is a Nash equilibrium.

The set $C_{1}\left(S^{n}\right)$ is a partition of the strategy set for player 1 while $C_{2}\left(S^{n}\right)$ is a partition of the strategy set for player 2. By Lemma 3.2(ii) a class $C_{1}(s)$ of player 1 "corresponds" to a class $C_{2}(-s)$ of player 2 in the sense that if player 1 puts probability 0 on all strategies in $C_{1}(s)$ then none of the strategies in $C_{2}(-s)$ are a best response for player 2 and vice versa. Part (i) of Lemma B.4 states that two corresponding classes contain the same number of elements. By (ii) for every class $C_{1}(s)$ there is always a Nash Equilibrium such that player 1's strategy has this class as its support while player 2's strategy has support $C_{2}(-s)$. The equilibrium constructed to prove (ii) is such that player 1 chooses his strategy (with support $C_{1}(s)$ ) such that player 2 is indifferent between all strategies in $C_{2}(-s)$ (and vice versa). As $\Gamma_{n}$ is a non-degenerate game, by Proposition 2(ii) this is the unique equilibrium $\left(\sigma_{1}, \sigma_{2}\right)$ such that $\operatorname{supp}\left(\sigma_{1}\right)=C_{1}(s)$ and $\operatorname{supp}\left(\sigma_{2}\right)=C_{2}(-s)$.

We are now ready to prove Theorem 2 and Proposition 4.

Proof of Theorem 2. If $\Gamma_{n}$ is a counter-circulant game in which player 1's payoff matrix is anticirculant and player 2's payoff matrix is circulant then by Lemma B.3, $C_{1}\left(S^{n}\right)$ and $C_{2}\left(S^{n}\right)$ as defined in section 3.1 are partitions of $S^{n}$. Further, by Lemma B.4(i), $\left|C_{1}\left(S^{n}\right)\right|=\left|C_{2}\left(S^{n}\right)\right|$ and by Lemmata 3.1(ii), 3.2(ii), and B.4(ii), $\Gamma_{n}, \bar{S}_{1}=C_{1}\left(S^{n}\right)$, and $\bar{S}_{2}=C_{2}\left(S^{n}\right)$ satisfy properties (a)-(c) in Proposition 5 and hence $\Gamma_{n}$ has $2^{\left|C_{1}\left(S^{n}\right)\right|}-1$ Nash equilibria. 
To prove (i)-(iii) it hence suffices to determine $\left|C_{1}\left(S^{n}\right)\right|$. Note that any class $C_{1}(s)$ contains either one or two elements. It contains one element if and only if $-s+k \equiv s$ which occurs if and only if either $2 s=k$ or $2 s=n+k$. Further, there are at most two singleton classes.

(i) If $n$ is odd, then either $n-k$ is odd (if $k$ is even) or $2 n-k$ is odd (if $k$ is odd). Hence there is one singleton class in $C_{1}\left(S^{n}\right)$ and since all other elements of $C_{1}\left(S^{n}\right)$ contain two elements, $\left|C_{1}\left(S^{n}\right)\right|=(n-1) / 2+1=(n+1) / 2$.

(ii) If both $n$ and $k$ are even, then both $k$ and $n+k$ are even and $k / 2,(n+k) / 2 \in S^{n}$. Hence there are two singleton classes in $C_{1}\left(S^{n}\right)$ and since all other elements of $C_{1}\left(S^{n}\right)$ contain two elements, $\left|C_{1}\left(S^{n}\right)\right|=(n-2) / 2+2=(n+2) / 2$.

(iii) If $n$ is even and $k$ is odd, then $n+k$ is odd and hence neither $k / 2 \in S^{n}$ nor $(n+k) / 2 \in S^{n}$. Hence there is no singleton class and hence all elements of $C_{1}\left(S^{n}\right)$ contain 2 elements, implying that $\left|C_{1}\left(S^{n}\right)\right|=n / 2=n / 2$.

If $\Gamma_{n}$ is a counter-circulant game in which player 1's payoff matrix is circulant and player 2's payoff matrix is anti-circulant, there is a permutation of row vectors that transforms player 1 's payoff matrix into an anti-circulant matrix. Applying the same permutation of row vectors to player 2's payoff matrix yields a different version of the same game in which strategies are differently labeled and player 1's payoff matrix is anti-circulant and player 2's payoff matrix is circulant (Lemma A.1(ii)). This permutation does not affect the number of Nash equilibria and hence the proof of Theorem 2 is complete.

Proof of Proposition 4. Note first that if player 1's payoff matrix is circulant then by Lemma A.1(i) the game can be transformed into a different version of the same game in which player 1's payoff matrix is anti-circulant by a permutation of row vectors. Since such a permutation does not affect the number of pure strategy Nash equilibria, we assume wlog that player 1's payoff matrix is anti-circulant.

(i) By Lemmata 3.1(ii) and B.4(ii), $\Gamma_{n}$ has one pure strategy Nash equilibrium if and only if one of the classes $C_{1}(s)$ is a singleton set, which by construction happens if and only if $n$ is odd.

(ii) By Lemmata 3.1(ii) and B.4(ii), $\Gamma_{n}$ has two pure strategy Nash equilibria if and only if two of the classes $C_{1}(s)$ are singleton sets, which by construction happens if and only if both $n$ and $k$ are even.

(iii) By Lemmata 3.1(ii) and B.4(ii), $\Gamma_{n}$ has no pure strategy Nash equilibrium if and only if none of the classes $C_{1}(s)$ is a singleton set, which by construction happens if and only $n$ is even and $k$ is odd.

Finally, we prove Lemma 3.3.

Proof of Lemma 3.3. (i) To see the first part, let $M=\bigcup_{j=1}^{m} I\left(s^{j}\right)$ be a union of elements of $I\left(S^{n}\right)$. By Lemma 3.1(i) and Lemma B.2, $\Gamma_{n}$ and $\bar{S}_{1}=\bar{S}_{2}=I\left(S^{n}\right)$ as defined in section 3.1 then satisfy the hypotheses of Proposition 5. Hence, there is a unique Nash equilibrium $\left(\sigma_{1}, \sigma_{2}\right)$ with $\operatorname{supp}\left(\sigma_{1}\right)=M$.

To prove the second part, let $\left(\sigma_{1}, \sigma_{2}\right)$ be a Nash equilibrium. By Lemma 3.1(i), $\operatorname{supp}\left(\sigma_{1}\right)$ is a union of elements in $I\left(S^{n}\right)$.

(ii) Too see the first part, let $M=\bigcup_{j=1}^{m} C_{1}\left(s^{j}\right)$ be a union of elements of $C_{1}\left(S^{n}\right)$. By Lemma B.3, $C_{1}\left(S^{n}\right)$ and $C_{2}\left(S^{n}\right)$ as defined in section 3.1 are partitions of $S^{n}$. Further, by Lemma B.4(i), 
$\left|C_{1}\left(S^{n}\right)\right|=\left|C_{2}\left(S^{n}\right)\right|$ and by Lemma 3.1(ii), Lemma 3.2(ii), and B.4(ii), $\Gamma_{n}, \bar{S}_{1}=C_{1}\left(S^{n}\right)$, and $\bar{S}_{2}=C_{2}\left(S^{n}\right)$ satisfy properties (a)-(c) in Proposition 5. It follows that there is a unique Nash equilibrium $\left(\sigma_{1}, \sigma_{2}\right)$ with $\operatorname{supp}\left(\sigma_{1}\right)=M$.

To prove the second part, let $\left(\sigma_{1}, \sigma_{2}\right)$ be a Nash equilibrium. By Lemma 3.1(ii), $\operatorname{supp}\left(\sigma_{1}\right)$ is a union of elements in $C_{1}\left(S^{n}\right)$. 
Appendix C: Tables 
Table 1: Examples of iso-circulant games.

Matching Pennies Rock-Paper-Scissors

$4 \times 4$ Coordination

Matrix Player 1

$$
\left(\begin{array}{rr}
1 & -1 \\
-1 & 1
\end{array}\right)
$$

$$
\left(\begin{array}{lll}
3 & 2 & 1 \\
2 & 1 & 3 \\
1 & 3 & 2
\end{array}\right)
$$$$
\left(\begin{array}{llll}
5 & 4 & 3 & 2 \\
4 & 3 & 2 & 5 \\
3 & 2 & 5 & 4 \\
2 & 5 & 4 & 3
\end{array}\right)
$$

Matrix Player 2

$$
\left(\begin{array}{rr}
-1 & 1 \\
1 & -1
\end{array}\right)
$$

$$
\left(\begin{array}{lll}
1 & 2 & 3 \\
2 & 3 & 1 \\
3 & 1 & 2
\end{array}\right)
$$

$\left(\begin{array}{llll}5 & 4 & 3 & 2 \\ 4 & 3 & 2 & 5 \\ 3 & 2 & 5 & 4 \\ 2 & 5 & 4 & 3\end{array}\right)$

Shift $k$

$\operatorname{gcd}(n, k)$

Number of Equilibria

$$
\begin{aligned}
& 1 \\
& 1 \\
& 1
\end{aligned}
$$

1
1
1

4
4
15

Equivalence Classes

$$
\begin{aligned}
& \mathrm{I}(0)=\{0\} \\
& \mathrm{I}(1)=\{1\}
\end{aligned}
$$

$$
\begin{aligned}
& \mathrm{I}(0)=\{0\} \\
& \mathrm{I}(1)=\{1\} \\
& \mathrm{I}(2)=\{2\}
\end{aligned}
$$$$
\mathrm{I}(0)=\{0\}
$$

Nash Equilibria

Pure

Support 2 Classes

$$
\begin{aligned}
& \sigma_{1}=(1 / 2,1 / 2) \\
& \sigma_{2}=(1 / 2,1 / 2)
\end{aligned}
$$

$$
\begin{aligned}
& s_{1}=0, s_{2}=0 s_{1}=1, s_{2}=3 \\
& s_{1}=2, s_{2}=2 \\
& s_{1}=3, s_{2}=1 \\
& \sigma_{1}=(1 / 4,3 / 4,0,0) \\
& \sigma_{2}=(3 / 4,0,0,1 / 4) \\
& \sigma_{1}=(1 / 2,0,1 / 2,0) \\
& \sigma_{2}=(1 / 2,0,1 / 2,0) \\
& \sigma_{1}=(3 / 4,0,0,1 / 4) \\
& \sigma_{2}=(1 / 4,3 / 4,0,0) \\
& \sigma_{1}=(0,1 / 4,3 / 4,0) \\
& \sigma_{2}=(0,0,1 / 4,3 / 4) \\
& \sigma_{1}=(0,1 / 2,0,1 / 2) \\
& \sigma_{2}=(0,1 / 2,0,1 / 2) \\
& \sigma_{1}=(0,0,1 / 4,3 / 4) \\
& \sigma_{2}=(0,1 / 4,3 / 4,0) \\
& \sigma_{1}=(1 / 4,1 / 4,1 / 2,0) \\
& \sigma_{2}=(1 / 2,0,1 / 4,1 / 4) \\
& \sigma_{1}=(1 / 4,1 / 2,0,1 / 4) \\
& \sigma_{2}=(1 / 4,1 / 2,0,1 / 4) \\
& \sigma_{1}=(1 / 2,0,1 / 4,1 / 4) \\
& \sigma_{2}=(1 / 4,1 / 4,1 / 2,0) \\
& \sigma_{1}=(0,1 / 4,1 / 4,1 / 2) \\
& \sigma_{2}=(0,1 / 4,1 / 4,1 / 2) \\
& \sigma_{2}=(1 / 3,1 / 3,1 / 3,1 / 3)=(1 / 4,1 / 4,1 / 4,1 / 4) \\
& \sigma_{1}=(1 / 4,1 / 4,1 / 4,1 / 4) \\
& \sigma_{2}=(1 / 3)
\end{aligned}
$$

Support 3 Classes 
Table 2: Examples of counter-circulant games.



